\title{
Dynamic shear stress in a double lap bonded assembly
}

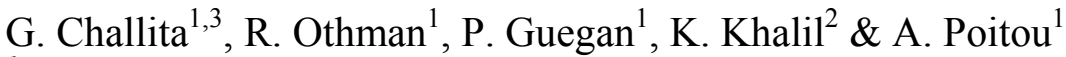 \\ ${ }^{1}$ Institut de Recherche en Génie Civil et Mécanique, Ecole Centrale de \\ Nantes, 1 Rue de la Noë, BP 92101,44321 Nantes Cedex 3, France \\ ${ }^{2}$ Département de Mécanique, Faculté de Génie Branche 1, \\ Université Libanaise, Tripoli, Liban \\ ${ }^{3}$ Département de Mécanique, Faculté de Génie Branche 2, \\ Université Libanaise, Roumieh, Liban
}

\begin{abstract}
This work consists of investigating the dynamic shear behaviour of adhesively bonded assemblies at high rates of loading. A double lap joint sample was adopted, such as the compressive wave, and transformed to a shear loading within the adhesive layer. The tool used for this target is the Split Hopkinson Pressure Bar (SHPB). A temperature of $20^{\circ} \mathrm{C}$ and hygrometry of $50 \%$ are the ambiance conditions used in the sample in order to avoid temperature and humidity effects. The adhesive material is the cyanoacrylate, while the adherent material is steel. The influence of high rates is remarkable on these bonded assemblies.
\end{abstract}

Keywords: Hopkinson bar, adhesively bonded assembly, shear, in-plane load, high strain rate.

\section{Introduction}

Bonded assemblies are becoming used very frequently and are widespread in industry, mainly in aircraft, automotive and electrical fabrications. Contrary to other ways of assembling, bonding has low costs, simplicity of manipulation and also ensures the uniformity of stress repartition on the bonded surfaces. In these applications, the adhesive joints can subdue impact loads as well as quasi-static loadings. However, the main goal of many researchers is to study the influence of the loading rate on the mechanical behaviour of adhesively bonded 
assemblies. Multiple quasi-static tests are specified in the ASTM standards [1]. Only a few investigations were carried out on the impact response of these assemblies. Kinloch and Kodokian [2] used the three-point bending specimen to study the fracture energy, while Sato [3] studied the absorbed energy in a CFRPaluminium alloy beam. Roy and Reddy [4] investigated the dependence of the resonance frequencies and loss factor on the adhesive shear modulus, lap ratio and strap thicknesses. Lawrence $\mathrm{Wu}$ et al. [5] measured the absorption energy in electronic adhesive joints using the Hopkinson bar technique. However, the investigation of adhesive joint strength did not constitute the topic of any of these studies.

Kaya [6] used the finite element method to investigate dynamic characteristics in an adhesively single lap joint under dynamic forces. Owen et al. [7] investigated the influence of surface roughness on shear joints. Harris et al. [8] measured the shear strength in a single-lap joint under an impact load. However, only a low-impact velocity of $1.34 \mathrm{~m} / \mathrm{s}$ is reached. Lataillade et al. succeeded in achieving higher impact velocities by using the Hopkinson bar technique (Lataillade et al. [9], Keisler and Lataillade [10]) and a tensile technique with an inertial wheel [11]. In line with [9], Yokoyama and Shimizu $[12,13]$ investigated the impact shear strength using a modified split Hopkinson bar by proposing a new geometry of the sample: a pin-and-collar specimen While Srivastava et al. [14] did a similar study with another new sample geometry: a double-L specimen. The impact velocity can reach $20 \mathrm{~m} / \mathrm{s}$ in these techniques. Different loading configurations were also investigated. For instance, the tensile strength of adhesive joints was investigated [15-19]. Moreover, the combined tension-torsion load was investigated by Sato and Ikegami $[20,21]$ using the Hopkinson bar technique.

In this paper, we are using the Hopkinson bar technique to determine the shear strength in the adhesively bonded joints. This technique takes into consideration the wave propagation in the experimental set-up. Since the specimen geometries proposed in the literature yield to an impedance mismatch with the bar, a new M-shaped specimen is proposed. It consists of a double lap adhesive joint.

The impedance mismatch has highly negative effects on the input and output force measurements: the incident wave, induced in the input bar by the strike of the projectile, reflects in this bar before reaching the adhesive joints. In the same way, the transmitted wave, through the adhesive joint, reflects before reaching the strain gauge cemented on the output bar.

\section{Theoretical study}

\subsection{Description of the Hopkinson bar apparatus}

Two elastic bars are the main components of the conventional Hopkinson bar apparatus [22], the first is the incident bar and the second is the transmitted bar; they are also known as input and output bars respectively (Fig. 1). A striker bar, whose length is less than the half that of the input bar, will move horizontally 
under $\mathrm{N}_{2}$ pressure of an air gun to hurt the first extremity of the input bar and thus to generate a compressive wave in this bar. This incident wave moves through the input bar until it reaches the bar-specimen interface. At this interface, the first part of the wave is reflected back into the input bar as a tensile wave and the second part is transmitted through the specimen into the output bar as a compressive wave. In order to measure the bars deformations, one gauge station is cemented on each bar. The input gauge station measures the incident and reflected waves while the output gauge records the transmitted wave. The input gauge is cemented in the middle of the input bar while the output gauge is positioned nearer to the specimen-output bar interface. In this case, the incident and the reflected waves are recorded separately with the input gauge. Assuming one-dimensional wave propagation, the forces applied by the bars on the specimen are given by:

$$
\begin{aligned}
F_{\text {in }}(t) & =A_{\text {in }} E_{\text {in }}\left(\varepsilon_{\text {inc }}(t)+\varepsilon_{\text {ref }}(t)\right) \\
F_{\text {out }}(t) & =A_{\text {out }} E_{\text {out }} \varepsilon_{\text {tra }}(t)
\end{aligned}
$$

where the subscripts in, out, inc, ref and tra mean input bar, output bar, incident wave, reflected wave and transmitted wave, respectively, and $A$ is the crosssection area, $E$ the Young's modulus, $\varepsilon$ is the wave's strain.

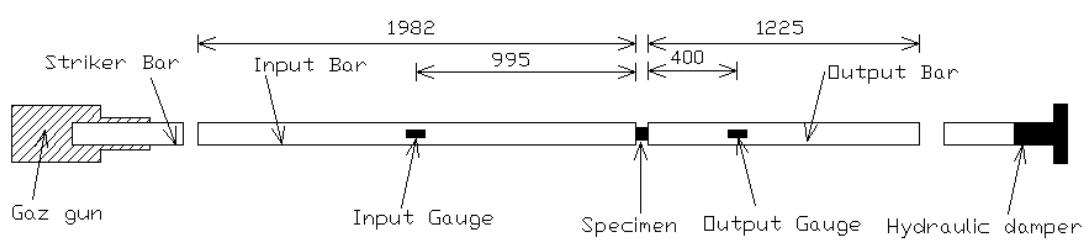

Figure 1: $\quad$ Simplified scheme of the Hopkinson bar apparatus.

\subsection{Specimen geometry}

The M-shaped specimen involves three adherent plates bonded by two adhesive layers (Fig. 2). The middle adherent plate is shifted from the other two. This gap will be useful to convert the compression loading into shear loading inside the adhesive layer. It is aligned with the output bar end while the lower and the upper plates will be aligned with the input bar end. The geometry of the specimen allows one to carry out all of the experiments on a classical Hopkinson Bar technique without any modification and thus avoiding any added impedance mismatch to the system. The mechanical impedance of each adherent plate is constant so as not to induce any undesired reflection of the waves. The in-plane movement of the bars is transformed by the geometry of the specimen to a shear loading on the adhesive layers. Assuming dynamic equilibrium in the specimen, the shear stress is calculated in the function of the input and output forces and the area of a single lap joint $\left(A_{\text {adh }}\right)$ as follows: 


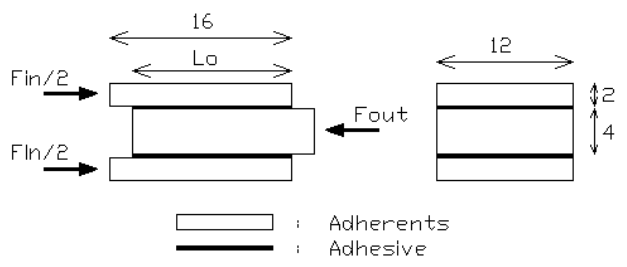

Figure 2: $\quad$ Specimen geometry: (a) side sight (b) rear sight.

$$
\tau(t)=\frac{F_{\text {in }}(t)+F_{\text {out }}(t)}{4 A_{\text {adh }}}
$$

\subsection{Bonding steps}

To ensure the good alignment of the specimen a special mounting device is developed (Fig. 3). To prepare the specimen, the following steps are followed:

1. The lower and middle adherent plates are wiped with dry paper.

2. The thickness of each plate is measured using a micrometer.

3. The mounting device is cleaned with acetone, while both plates are immersed in acetone to be treated by ultrasound waves for one minute.

4. A hairdryer is used to dry the plates.

5. The adhesive is spread on the upper face of the lower plate, this plate is then fixed by a screw in the mounting device as shown in Fig. $3 a$.

6. The adhesive is spread on the lower face of the middle plate, this plate is then fixed by a screw in the mounting device on the opposite side to the first screw to create the gap between the two plates, and than both plates are fixed by a vertical screw to keep constant pressure as shown in Fig. $3 b$.

7. The assembly is kept at room conditions for 5-6hours

8. The upper plate is treated similarly to the previous plates as mentioned above.

9. The vertical screw is unscrewed, the adhesive is spread on the upper face of the middle plate and on the lower face of the upper plate and this plate is fixed by a screw in the mounting device on the same side as the first screw, as shown in Fig. 3c.

10. The whole specimen is then fixed in the mounting device by a vertical pressure screw to keep a constant pressure and ensure a convenient adhesion between the three plates, as shown in Fig. 3d.

11. The specimen is cured at room temperature for 18 hours.

12. The specimen is kept in a conditioned room (local temperature $20^{\circ} \mathrm{C}$, relative hygrometry $50 \%$ ) for at least one week.

13. The specimen is tested at room temperature after no more than two hours from getting it out from the conditioned room. 


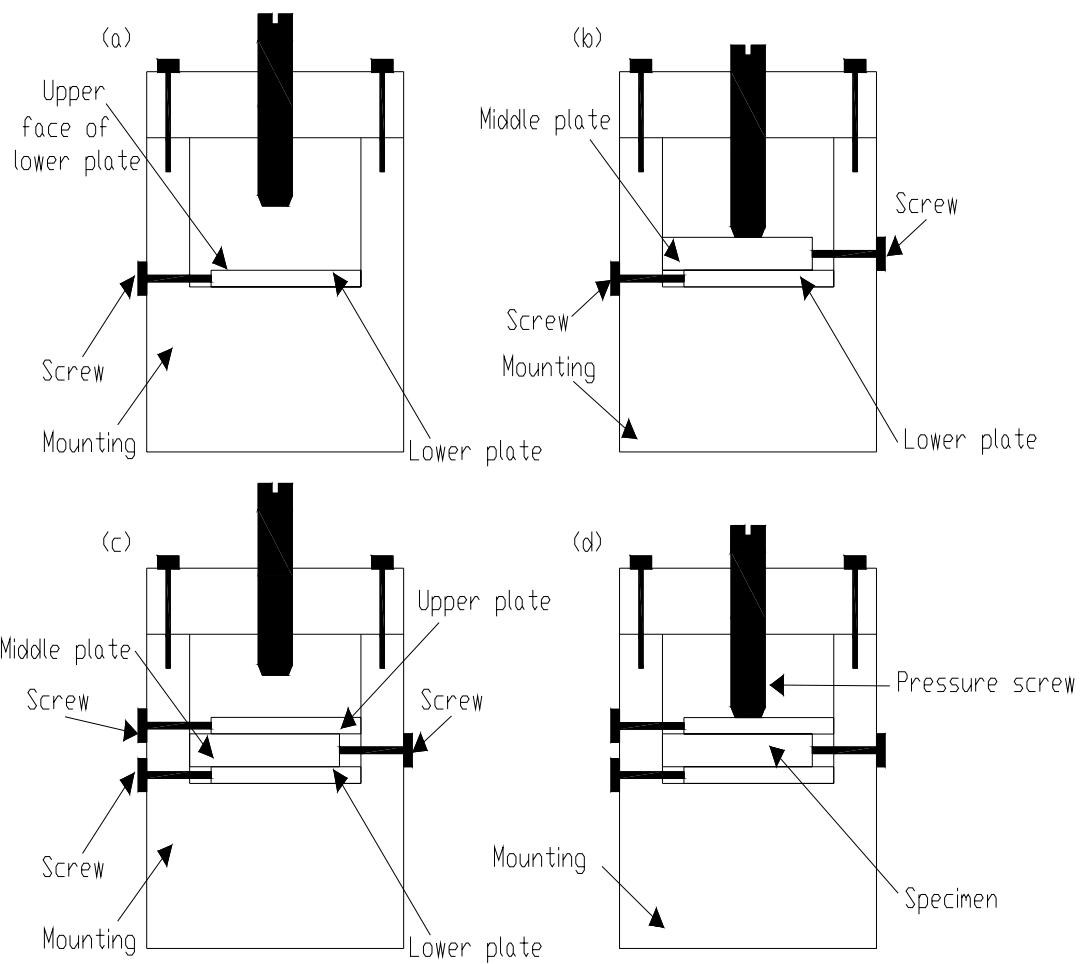

Figure 3: $\quad$ Specimen preparation, bonding steps.

\section{Experimental study}

The input and output Hopkinson bars are both made from steel material (Fig. 1). Their mechanical properties are suitable for high rates of loading. We present in the following all the engineering properties in the experiments:

- The bars' diameter is $16 \mathrm{~mm}$.

- The bars' Young's modulus is 190GPa.

- The bars' elastic limit is $1400 \mathrm{MPa}$.

- The specimen's adherent plates are made of steel (35NCD16).

- The three adherent plates are bonded with a cyanoacrylate based adhesive (2610).

- The adhesive layer thickness is $20 \mu \mathrm{m} \pm 8 \mu \mathrm{m}$.

- The strain measurements are recorded at a frequency rate of $10 \mathrm{MHz}$.

The software DAVID [23], developed at the Ecole Polytechnique (France), is used to treat the deformations' signals recorded by the stain gauges and then to 
compute the values of the forces at both interfaces specimen bars. Thus, the shear strength is deduced using Eq. (2).

On the other hand, comparison is carried out between the impact shear strength and a conventional quasi-static machine, where tests were applied to this M-shaped specimen. Fig. 4 shows the variation of the shear strength with the mean value of the shear rate. Since a bilinear behaviour is observed, the shear strength is found to be highly rate-sensitive. As the graph shows, at rates greater than $5.10^{5} \mathrm{MPa} \mathrm{s}-1$, the gradient increases considerably. Two joint overlap lengths $\left(l_{j}\right.$ : see Fig. 2$)$ are tested $(9$ and $14 \mathrm{~mm})$. The results obtained with the short specimens confirm the bilinear behaviour.

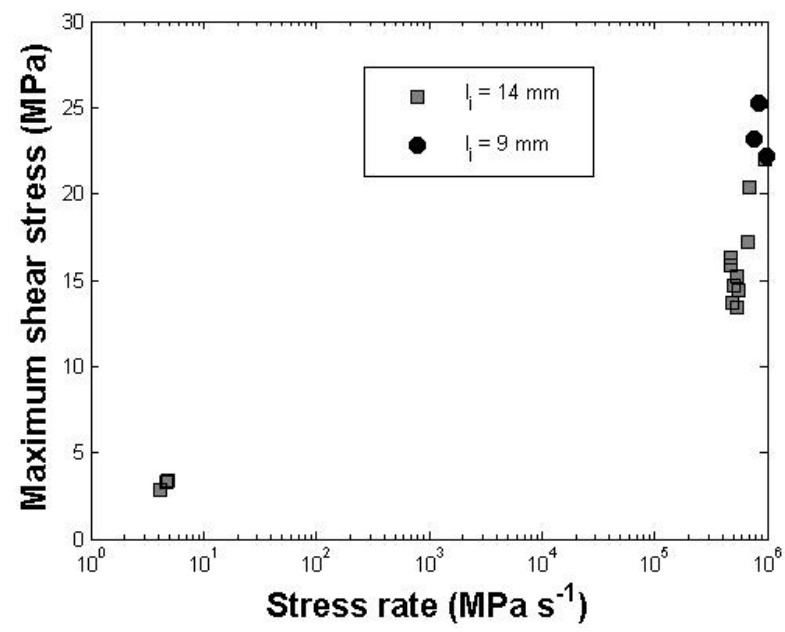

Figure 4: $\quad$ Variation of the shear strength with the loading rate.

\section{Conclusion}

A new sample geometry was presented in this paper. Its advantage is that it minimizes the impedance mismatch with the bar. The influence of the loading rate on the shear strength of double-lap adhesive joints was investigated using the Hopkinson bar technique. It was to be found high-rate sensitive: a bilinear behaviour is observed. Moreover, a mounting device is developed to ensure an accurate alignment between the specimen and the two bars.

\section{References}

[1] 1995 Annual Book of ASTM Standards, Vol. 15.06, adhesives, ASTM, (1995), Philadelphia.

[2] A. L. Kinloch, G. A. Kodokian, Journal of adhesion, Vol. 24, (1987), pp. 109.

[3] C. Sato, Journal de Physique IV, Vol. 110, (2003), pp. 747-752. 
[4] S. Roy, N. Reddy, Finite element models of viscoelasticity and diffusion in adhesively bonded joints, International Journal for Numerical Methods in engineering 26 (1988) 2531.

[5] C.M. Lawrence Wu, R.K.Y. Li, N.H. Yeung, Journal of Electronic Packaging, Transactions of the ASME, Vol. 125, (2003), pp. 93-97.

[6] A. Kaya, Investigation of stress distribution in adhesive-bonded lap joint, MS Degree Thesis, Dokuz Eylul University, Institute of Science and Technology, December, Izmir, 1991.

[7] Owens JP, Lee-Sullivan P. Int. J Adhes 2000; 20:39-45.

[8] J. A. Harris, Proceedings of the institute of mechanical engineering, Vol. 199, No. C2, (1985).

[9] J.L. Lataillade, C. Keisler, Ph. Charobonnet, Preprints of EURADH'92 Conference (Karlsruhe, Germany), (1992), pp. 584-589.

[10] C. Keisler, J.L. Lataillade, Journal of Adhesion Science and Technology, Vol. 9, (1995), pp. 395-411.

[11] F. Cayssials, J.L. Lataillade, Journal of adhesion, Vol. 56, (1996), pp. 281.

[12] T. Yokoyama, Key of Engineering Materials, Vols. 145-149, (1998), pp. 317-322.

[13] T. Yokoyama, H. Simizu, JSME International Journal, Series A, Vol. 41, (1998), pp. 503-509.

[14] Srivastava, A. Shukla, V. Parameswaran, Journal of Testing and Evaluation, Vol. 28, (2000), pp. 438-442.

[15] T. Yokoyama, International Conference on advanced Technology in Experimental Mechanics, Vol. 2, 21-24 July 1999, pp. 366-371.

[16] T. Sawa, Y. Suzuki, I. Higuchi, Impact Engineering and Application. Proceedings of the $4^{\text {th }}$ International Symposium on Impact Engineering. Ed.

A. Chiba \& S. Tanimura. Elsevier Science Ltd., (2001), pp. 469-574.

[17] T. Yokoyama, Journal of strain analysis for engineering design, Vol. 38, (2003), pp. 233-245.

[18] T. Yokoyama, K. Nakai, Journal de Physique IV, Vol. 134, (2006), pp. 789-795.

[19] H. Wada, K. Suzuki, K. Murase, T.C. Kennedy, Impact Engineering and Application. Proceedings of the $4^{\text {th }}$ International Symposium on Impact Engineering. Ed. A. Chiba \& S. Tanimura. Elsevier Science Ltd., (2001), pp. 463-468.

[20] C. Sato, K. Ikegami, Journal of adhesion, Vol. 70, (1999), 57-73.

[21] C. Sato, K. Ikegami, American Society of Mechanical Engineers, Design Engineering Division, Vol. 105, (1999), 139-143.

[22] H. Kolsky, Proceedings of the Physical Society B, Vol. 62, (1949), pp. 676700 .

[23] G. Gary, V. DeGreff, DAVID User Manual. 